\title{
ORIGINAL ARTICLE OTUB1 inhibits the ubiquitination and degradation of FOXM1 in breast cancer and epirubicin resistance
}

\author{
U Karunarathna ${ }^{1,6}$, M Kongsema ${ }^{1,6}$, S Zona ${ }^{1,6}$, C Gong $^{1,2}$, E Cabrera ${ }^{3}$, AR Gomes ${ }^{1}$, EPS Man ${ }^{2}$, P Khongkow ${ }^{1}$, JW-H Tsang ${ }^{4}$, U-S Khoo ${ }^{2}$, \\ $\mathrm{RH}$ Medema ${ }^{5}$, R Freire ${ }^{3}$ and EW-F Lam ${ }^{1}$
}

The forkhead transcription factor FOXM1 has a key role in DNA damage response, and its deregulated overexpression is associated with genotoxic drug resistance in breast cancer. However, little is known about the posttranslational mechanisms by which FOXM1 expression is regulated by genotoxic agents and how they are deregulated in resistant cells. Initial co-immunoprecipitation studies verified previous proteomic analysis finding that the OTUB1 is a novel FOXM1-interacting protein. Western blot analysis showed that both OTUB1 and FOXM1 expression reduced upon genotoxic agent treatment in MCF-7 cells, but remained relatively constant in resistant cells. FOXM1 expression reduced upon OTUB1 depletion by siRNA and increased with OTUB1 overexpression in MCF-7 cells, arguing that OTUB1 positively regulates FOXM1 expression. In agreement, co-immunoprecipitation experiments demonstrated that FOXM1 expression is associated with OTUB1 binding but inversely correlates with conjugation to the protein degradation-associated Lys-48-linked ubiquitin-chains. Overexpression of wild-type (WT) OTUB1, but not the OTUB1(C91S) mutant, disrupted the formation of Lys48-linked ubiquitin-conjugates on FOXM1. Importantly, knockdown of OTUB1 by siRNA resulted in an increase in turnover of FOXM1 in MCF-7 cells treated with the protein synthesis inhibitor cycloheximide, whereas overexpression of WT OTUB1, but not the OTUB1(C91S) mutant, significantly enhances the half-life of FOXM1. In addition, proliferative and clonogenic assays also show that OTUB1 can enhance the proliferative rate and epirubicin resistance through targeting FOXM1, as OTUB1 has little effect on FOXM1-deficient cells. The physiological relevance of the regulation of FOXM1 by OTUB1 is further underscored by the significant correlations between FOXM1 and OTUB1 expression in breast cancer patient samples. Cox-regression survival analysis indicates that OTUB1 overexpression is linked to poorer outcome in particular in patients treated with chemotherapy. Collectively, these data suggest that OTUB1 limits the ubiquitination and degradation of FOXM1 in breast cancer and has a key role in genotoxic agent resistance.

Oncogene (2016) 35, 1433-1444; doi:10.1038/onc.2015.208; published online 6 July 2015

\section{INTRODUCTION}

Breast cancer is one of the most prevalent causes of death in women worldwide. Genotoxic anti-cancer agents, including anthracyclines, platinum compounds, methylating agents and ionizing irradiation, are used widely to treat breast cancer patients who are not suitable for hormonal therapy and those with advanced or metastatic cancer. These genotoxic agents are also often used in the adjuvant setting, particularly after surgery, to prevent the return of the disease. However, resistance to these agents often emerges in patients, and this leads to suboptimal efficacy and disease relapse. ${ }^{1}$ The cellular response to DNA damage is a key determinant of the efficacy of these genotoxic agents, and these reactions include initiation of DNA damage repair response, cell cycle-checkpoint activation and induction of apoptosis or senescence. These processes ultimately govern cell fate and sensitivity to radiotherapy or chemotherapy. Conversely, a deregulated DNA damage response can lead to resistance to these anticancer agents.

Substantial evidence has accumulated to indicate that the Forkhead box M1 (FOXM1) transcription factor has a central role in cell proliferation, migration, invasion, angiogenesis, stem cell renewal, DNA damage repair and cellular senescence, which impact tumour initiation, progression, metastasis, angiogenesis and drug sensitivity. Recent research also indicates that deregulated FOXM1 overexpression confers genotoxic and other chemotherapeutic agent resistance in cancer. ${ }^{2-7}$ There is already good evidence that FOXM1 acts as a mediator of DNA damage response as well as a modulator of genotoxic agent sensitivity. ${ }^{4-6,8-10}$ Even though deregulated FOXM1 overexpression is considered key to the development of genotoxic agent resistance, the specific mechanisms involved in FOXM1 deregulation remain unknown. Therefore, a better understanding of the mechanisms that regulates FOXM1 expression in response to genotoxic agents and how FOXM1 is deregulated in resistant cancer cells is of importance for designing new therapeutic approaches directed to this degradation pathway.

Epirubicin is an anthracycline genotoxic drug commonly used for treating breast cancer. ${ }^{11}$ FOXM1 is downregulated by epirubicin at the transcriptional levels in breast cancer cells. $^{8,12,13}$ However, the fact that FOXM1 protein expression

\footnotetext{
${ }^{1}$ Department of Surgery and Cancer, Imperial College London, Hammersmith Hospital Campus, London, UK; ${ }^{2}$ Department of Pathology, Li Ka Shing Faculty of Medicine, The University of Hong Kong, Hong Kong SAR, China; ${ }^{3}$ Unidad de Investigación, Hospital Universitario de Canarias, Instituto de Tecnologías Biomédicas, Ofra s/n, La Laguna, Tenerife, Spain; ${ }^{4}$ Department of Clinical Oncology, Li Ka Shing Faculty of Medicine, The University of Hong Kong, Hong Kong SAR, China and ${ }^{5}$ Division of Cell Biology, The Netherlands

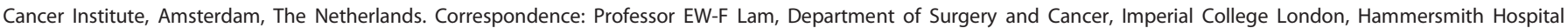
Campus, Room 134, 1st Floor, Imperial Centre for Translational and Experimental Medicine (ICTEM), Hammersmi, Du Cane Road, W12 0NN London, UK.

E-mail: eric.lam@imperial.ac.uk

${ }^{6}$ These authors contributed equally to this work.

Received 27 March 2015; revised 28 April 2015; accepted 14 May 2015; published online 6 July 2015
} 
declines at a faster kinetics when compared with its mRNA transcripts in response to genotoxic agents indicates that posttranscriptional mechanisms have a central role in regulating its DNA-damaging agent response in breast cancer cells., ${ }^{8,12}$ In agreement, we have also shown recently that upon epirubicin treatment, FOXM1 is modified through SUMOylation, which leads to its ubiquitination and degradation through the ubiquitinproteasome proteolytic pathway. ${ }^{10}$

Ubiquitination is a posttranslational modification that confers a range of protein regulatory functions, including targeting a substrate protein for degradation, modifying its activity, adjusting its function, changing its subcellular location and altering proteinprotein interactions. Ubiquitination is a dynamic process and can be reversed by deubiquitination, which is catalysed by a family of proteins that are ubiquitin hydrolases or deubiquitinating enzymes called DUBs. OTUB1 (OTU domain-containing ubiquitin aldehyde-binding proteins 1; also called Otubain 1) belongs to the ovarian tumour domain protease (OTU) subfamily of DUBs. OTUB1 can negatively regulate ubiquitination and control protein stability and activity via a non-canonical mechanism as well as the conventional catalytic process. More specifically, OTUB1 can function as a cysteine protease that hydrolyses the isopeptide bond between ubiquitin and the target protein or another ubiquitin molecule. Alternatively, OTUB1 can also operate through a non-canonical mechanism independently of its catalytic activity, by inhibiting the activity of E2 ubiquitin-conjugating enzymes.

To explore the mechanisms by which FOXM1 expression is modulated at the posttranslational level as well as the molecules involved, we performed mass spectrometry analysis of protein complexes co-immunoprecipitated with FOXM1 in the breast carcinoma MCF-7 cell line and identified the deubiquitinase OTUB1 as a novel FOXM1-interacting factor. Given our previous finding that genotoxic agents cause FOXM1 SUMOylation, ubiquitination and degradation, these observations led us to hypothesize that OTUB1 promotes FOXM1 expression at the posttranslational level to potentiate genotoxic agent resistance. In this study, we examined the potential role of OTUB1 in the regulation of FOXM1 expression in genotoxic drug-sensitive and -resistant breast cancer cells.

\section{RESULTS}

OTUB1 interacts with FOXM1 in breast cancer cells

To confirm the mass spectrometry-based proteomic finding, we first examined whether endogenous OTUB1 binds to FOXM1 in the drug-sensitive MCF-7 and the drug-resistant MCF-7Epi ${ }^{R}$ cells by co-immunoprecipitation. In these cell extracts, we detected FOXM1 after immunoprecipitation with an OTUB1 antibody and vice versa, but not with the control lgG, indicating that OTUB1 interacts with FOXM1 either directly or as part of a larger complex. In addition, the co-immunoprecipitation studies also revealed that the FOXM1-OTUB1 complexes were more abundant in the MCF-7Epi ${ }^{R}$ compared with MCF-7 cells (Figures $1 a$ and $b$ and Supplementary Figure S1a). The FOXM1-OTUB1 interaction was further confirmed by the ability of FOXM1 to co-precipitate with a transiently transfected Flag-tagged OTUB1 (Supplementary Figure $\mathrm{S} 1 \mathrm{~b})$. Epirubicin treatment caused a rapid decrease in the FOXM1OTUB1 interaction in the sensitive MCF-7 cells but the levels of FOXM1-OTUB1 complexes were largely maintained or increased in the resistant MCF-7Epi ${ }^{R}$ cells (Figures $1 c$ and d; also see Figure 3 ). Given that OTUB1 is a deubiquitinating enzyme that removes polyubiquitin chains and promotes stability of target proteins, ${ }^{14-16}$ these data suggest that OTUB1 binds to FOXM1 and modulates its ubiquitination and degradation in response to genotoxic treatment in breast cancer.
OTUB1 regulates FOXM1 expression in response to epirubicin in breast cancer cells

To explore the potential regulation of FOXM1 by OTUB1 in response to genotoxic agents, we investigated the expression of OTUB1 and FOXM1 in the epirubicin-sensitive MCF-7 and epirubicin-resistant MCF-7Epi ${ }^{R}$ breast carcinoma cell lines following epirubicin treatment. Western blot results showed that both OTUB1 and FOXM1 expressed at higher levels in the resistant MCF-7Epi ${ }^{\mathrm{R}}$ cells compared with the parental MCF-7 cells. Moreover, both OTUB1 and FOXM1 decreased in expression levels in response to epirubicin in the sensitive cells, but were moderately induced in the resistant cells (Figure 2a). Consistently, the expression of the FOXM1 downstream target cyclin B1 also displayed a similar expression pattern as FOXM1 and OTUB1. Furthermore, epirubicin treatment also induced the accumulation of cleaved-PARP, indicative of apoptosis, in the sensitive MCF-7 but not in the resistant MCF-7Epi ${ }^{R}$ cells, confirming the differential drug sensitivity of these MCF-7 cells. Tubulin was also immunoblotted for as a control to ensure equal loading. Next, we studied the expression of OTUB1 and FOXM1 in response to another DNAdamaging agent, $\gamma$-irradiation. To this end, MCF-7 and MCF-7Epi ${ }^{R}$ cells were harvested for western blot analysis at $24 \mathrm{~h}$ after exposure to various doses of $\gamma$-irradiation (Figure $2 \mathrm{~b}$ ). The results again revealed that the expression levels of OTUB1 and FOXM1 were higher in the resistant compared with the sensitive MCF-7 cells. Although $\gamma$-irradiation caused an induction in OTUB1 and a corresponding increase in FOXM1 expression in a dose-dependent manner in the MCF-7Epi ${ }^{\mathrm{R}}$ cells, the induction of OTUB1 by $\mathrm{Y}^{-}$ irradiation were not matched by a similar induction of FOXM1 expression in the MCF-7 cells, which likely reflects the low baseline levels of OTUB1 in MCF-7 cells. Nevertheless, the correlations between the kinetics of OTUB1 and FOXM1 expression in the sensitive and resistant MCF-7 cells in response to DNA-damaging agents support the notion that OTUB1 restricts the downregulation of FOXM1 expression in response to DNA damage and genotoxic agents.

FOXM1 expression is positively regulated by OTUB1 and negatively by the proteasome pathway

To investigate the idea that OTUB1 modulates FOXM1 expression further, we examined the effects of silencing OTUB1 and FOXM1 on the expression of endogenous OTUB 1 and FOXM1 protein in MCF-7 cells. The results showed that in MCF-7 cells OTUB1 depletion culminated in the downregulation of FOXM1, but not vice versa (Figure $2 \mathrm{C}$ ), suggesting that OTUB1 positively regulates FOXM1 expression. In agreement, OTUB1 overexpression also caused an increase in FOXM1 levels (Figure 2d). It is noteworthy that depletion of FOXM1 had little effect on OTUB1 mRNA levels (Supplementary Figure S2). Together these results suggest that OTUB1 regulates FOXM1 expression primarily at the translational or posttranslational levels (Figure $2 \mathrm{~d}$ ). The results also indicate that OTUB1 has a role in modulating the steady-state level of the FOXM1 protein.

Given that OTUB1 has been shown to restrict the ubiquitination and proteasome degradation of target proteins in response to DNA damage, ${ }^{17}$ we asked whether FOXM1 is also downregulated through protein degradation by the ubiquitin-proteasome pathway and tested the effect of the proteasome inhibitor MG132 treatment on the expression of endogenous FOXM1 in the absence or presence of epirubicin in MCF-7 cells (Figure 2e). Treatment with MG132 essentially prevented the downregulation in FOXM1 expression levels by epirubicin, indicating that the downregulation of FOXM1 expression in response to epirubicin is, at least partially, due to proteasomal degradation. It is also notable that MG132 enhanced the expression of FOXM1 in untreated cells, suggesting that FOXM1 is continuously downregulated by proteasomal degradation at the steady state level. 

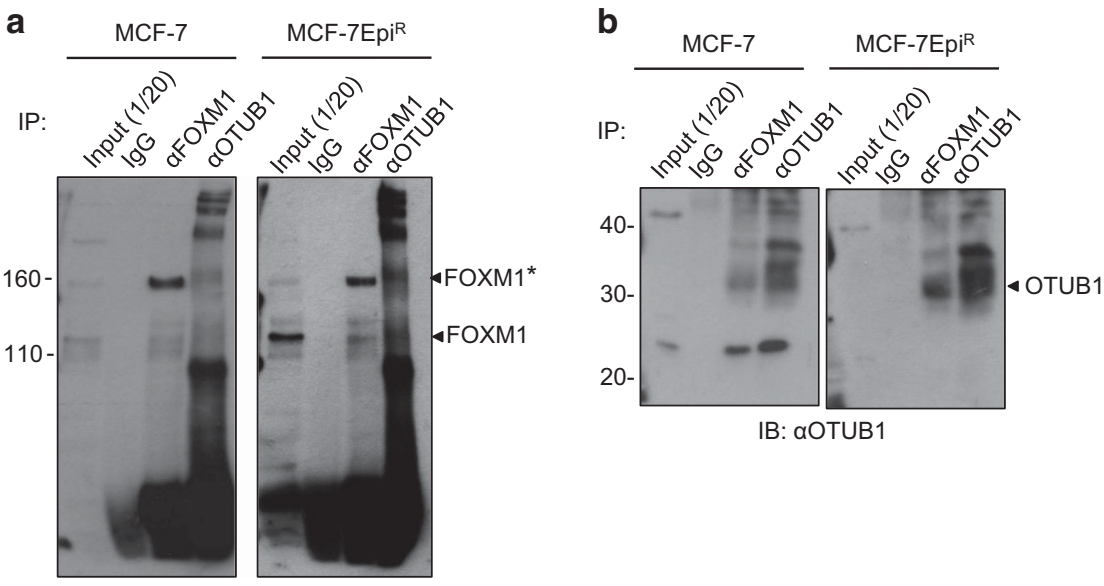

IB: aFOXM1

C

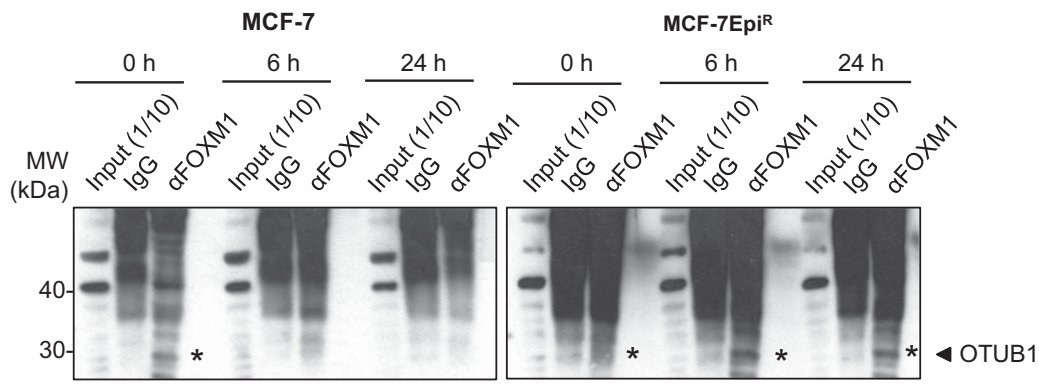

IB: $\alpha$ OTUB1

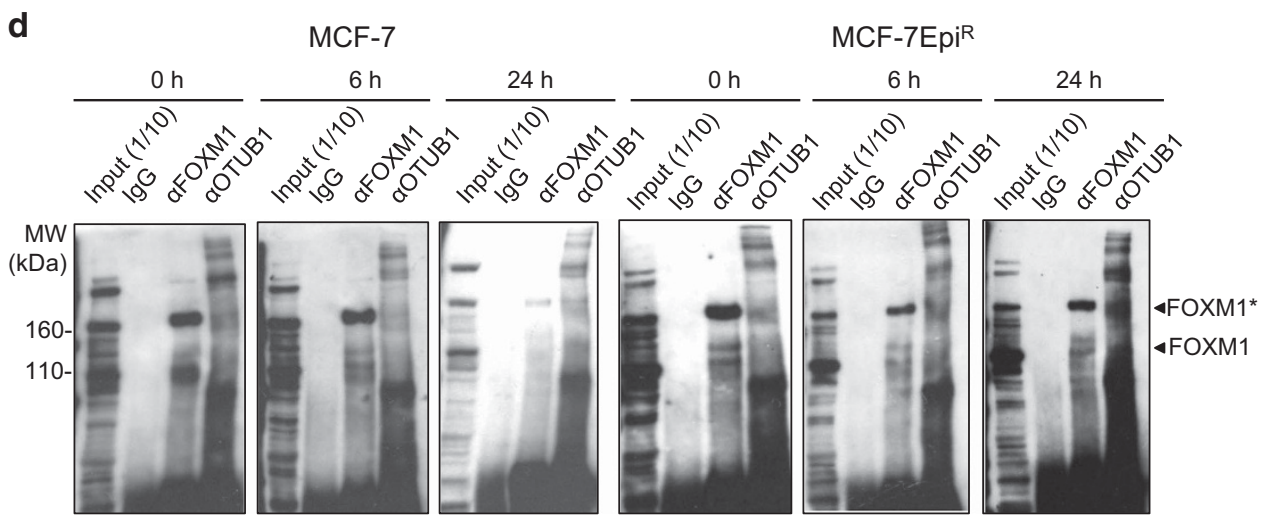

IB: aFOXM1

Figure 1. FOXM1 complexes with OTUB1 in MCF-7 and MCF-7Epi ${ }^{\mathrm{R}}$ cells. Co-immunoprecipitation (co-IP) was performed with an IgG antibody control, a FOXM1 $\left(\alpha\right.$ or anti-FOXM1) or an OTUB1 ( $\alpha$ OTUB1) antibody on lysates from MCF-7 and MCF-7Epi ${ }^{R}$ cells; Inputs (1/20 of IP), and IP products with IgG and specific antibodies were resolved on western blot and probed for (a) FOXM1 and (b) OTUB1. FOXM1* represents a FOXM1 species associated with its SUMOylation. (c) MCF-7 and MCF-7Epi ${ }^{\mathrm{R}}$ cells were treated with epirubicin $(1 \mu \mathrm{M})$ for 0,6 and $24 \mathrm{~h}$. Co-IP was performed with an IgG antibody control and a FOXM1 antibody ( $\alpha$ FOXM1); Inputs (1/10 of IP), and IP products with IgG and a FOXM1 antibody $(\alpha F O X M 1)$ were resolved on western blot and probed for OTUB1. (d) Lysates prepared from epirubicin-treated MCF-7 and MCF-7Epi ${ }^{R}$ cells as in (c) were precipitated with an IgG antibody control, a FOXM1 ( $\alpha$ FOXM1) and an OTUB1 ( $\alpha$ OTUB1) antibody and probed for FOXM1 expression.

OTUB1 binding promotes FOXM1 expression and attenuates Lys-48 linked ubiquitination in MCF-7 breast cancer cells We next examined whether OTUB1 binding is associated with the suppression of FOXM1 ubiquitination and downregulation in the epirubicin-sensitive and -resistant MCF-7 cells. To this end, we studied by co-immunoprecipitation the levels of OTUB1 and ubiquitination chains associated with FOXM1 in MCF-7 and $M C F-7^{R}$ cells in response to epirubicin (Figure $3 a$ and Supplementary Figure S3). The result showed that epirubicin treatment caused a prominent decrease in FOXM1 levels, which was associated with decreased interactions with OTUB1 in the sensitive MCF-7 cells. By contrast, FOXM1 protein levels and its association with OTUB1 remained relatively constant in the resistant cells upon epirubicin treatment. There was also an apparent loss of polyubiquitin chains in the FOXM1 complexes upon epirubicin treatment in the MCF-7 cells, but this is likely the result of a decline in endogenous FOXM1 levels upon epirubicin treatment. However, when we quantified the FOXM1 and Lys-48-linked polyubiquitin conjugates from the same immunoprecipitates, we found that the relative levels of Lys-48-linked polyubiquitin chains co-precipitated with FOXM1 actually increased with epirubicin treatment in MCF-7 cells (Figure 3b; Supplementary Figure S4). This induction in 

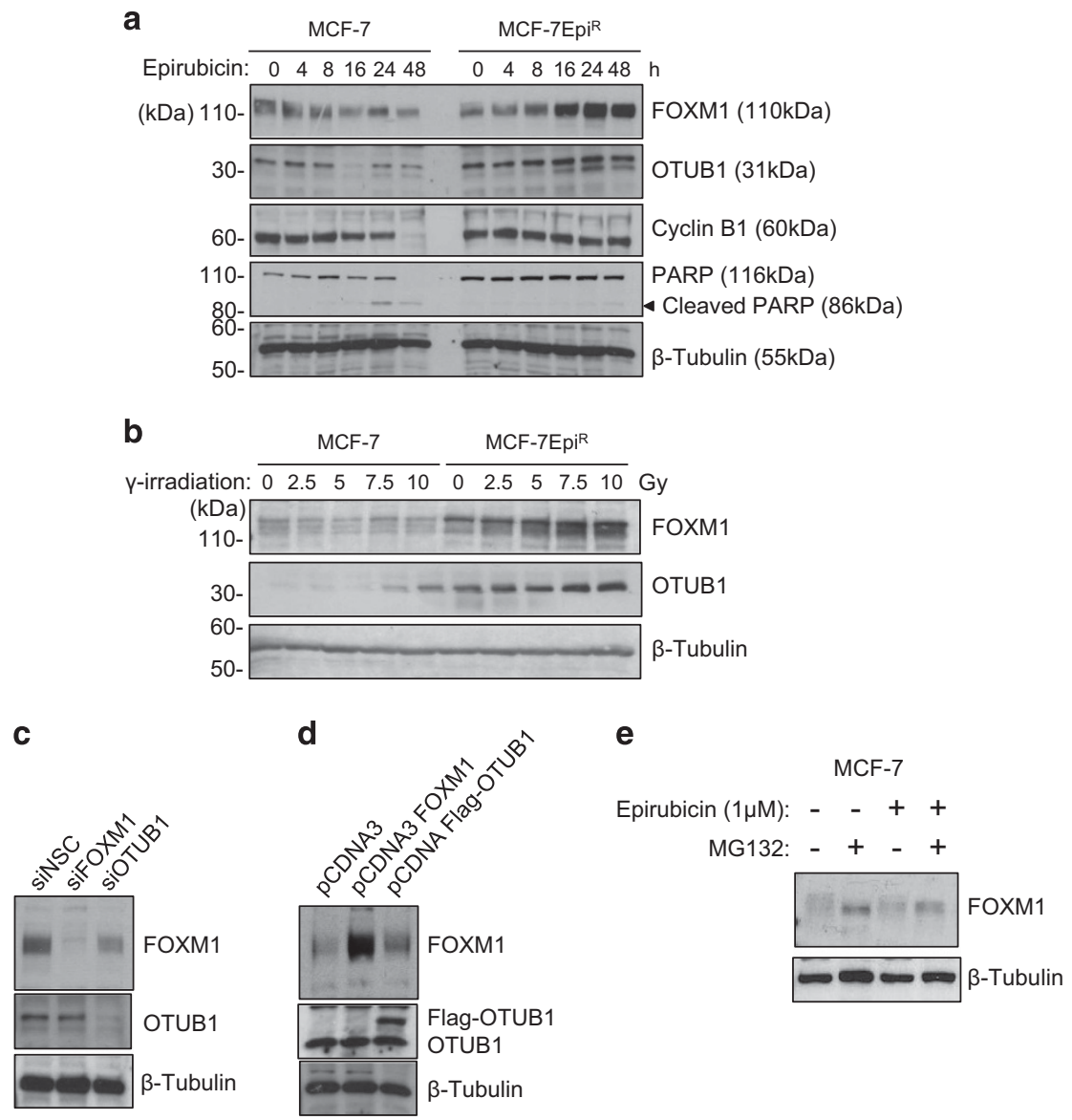

Figure 2. FOXM1 correlates with OTUB1 expression in MCF-7 and MCF-7Epi ${ }^{\mathrm{R}}$ cells. (a) Protein expression levels of FOXM1, OTUB1, Cyclin B1 and PARP in MCF-7 and MCF-7Epi ${ }^{\mathrm{R}}$ cell lines were examined by western blotting after epiribicin treatment at different time points indicated. $\beta$-Tubulin was probed as a control for protein loading. Notably, there was cleavage of PARP in the sensitive and not resistant cells upon epirubicin treatment. (b) Protein expression levels of FOXM1 and OTUB1 in MCF-7 and MCF-7Epi ${ }^{\mathrm{R}}$ cell lines were examined by western blotting with specific antibodies as indicated $24 \mathrm{~h}$ after treatment with different doses of $\gamma$-irradiation as indicated. (c) The expression levels of FOXM1, OTUB1 and $\beta$-tubulin were analysed by western blot analysis in MCF-7 cells transfected with the non-silencing control (NSC) siRNA, siRNA pool against FOXM1 or siRNA pool targeting OTUB1. (d) The expression levels of FOXM1, OTUB1 and $\beta$-tubulin were examined by western blot analysis in MCF-7 cells transfected with the empty expression vector pcDNA3, pcDNA3-FOXM1 or pcDNA3-Flag-tagged OTUB1. (e) The expression levels of FOXM1 and $\beta$-tubulin were examined by western blot analysis in MCF-7 cells treated with the proteasome inhibitor MG132 in the presence or the absence of epirubicin for $24 \mathrm{~h}$.

Lys48-linked polyubiquitination by epirubicin in MCF-7 cells was also associated with a decline in the FOXM1-OTUB1 interaction and FOXM1 expression in the MCF-7 cells. Quantification of the immunoblot signals indicated the kinetics for the disassociation of FOXM1-OTUB1 complexes occurred at a faster rate than the downregulation of FOXM1 expression, indicating that OTUB1 disassociation precedes FOXM1 ubiquitination and degradation in response to epirubicin in MCF-7 cells (Figure 3b). By comparison, the relative levels of total and Lys48-linked polyubiquitin chains, FOXM1 protein and FOXM1-OTUB1 complexes remained relatively constant in the resistant cells. It is noteworthy that the kinetics for Lys63linked polyubiquitin downregulation was similar to that of FOXM1 expression, indicating that the Lys63-linked polyubiquitin chains are less likely to be involved in FOXM1 degradation in response to epirubicin (Supplementary Figure S5). These results are consistent with the notion that OTUB1 binds to FOXM1 and limits its ubiquitination and degradation in MCF-7 breast cancer cells.

Polyubiquitin chains conjugated to FOXM1 are suppressed by OTUB1

We next investigated whether the polyubiquitin chains detected are directly conjugated to FOXM1. To confirm direct covalent linkage of Ubiquitin to FOXM1, we expressed a GFP-FOXM1 in the presence or absence of a His-tagged Ubiquitin, and pulled down the His-tagged ubiquitinated proteins under denaturing conditions after incubation with the proteasome inhibitor MG132 (Figure 4a). The purified His-tagged Ubiquitin-conjugated proteins were then immunoblotted with an anti-FOXM1 antibody, which detected the Ubiquitin-conjugated forms of FOXM1 as smears above the predicted GFP-FOXM1 molecular weight of $160 \mathrm{kDa}$, indicative of polyubiquitin chains. We next studied by co-immunoprecipitation whether overexpression of OTUB1 can repress the conjugation of K48-linked polyubiquitin chains, which are known to promote target protein degradation, on FOXM1 in MCF-7 cells. The results showed that the FOXM1 immunoprecipitates contained higher levels of Lys48-linked polyubiquitin chains in both untreated and epirubicin-treated MCF-7 cells when compared with the epirubicin-resistant MCF-7Epi ${ }^{\mathrm{R}}$ cells and two MCF-7 cell lines, one stably overexpressing OTUB1 and the other Flag-OTUB1 (Figure 4b and Supplementary Figure S6). The results also demonstrated that the levels of Lys48-linked polyubiquitin conjugates associated with FOXM1 increased upon epirubicin treatment. We next investigated whether the deubiquitinase activity of OTUB1 is required for FOXM1 stabilization and K48-polyubiquitin chain formation. To achieve this, we transfected 
a

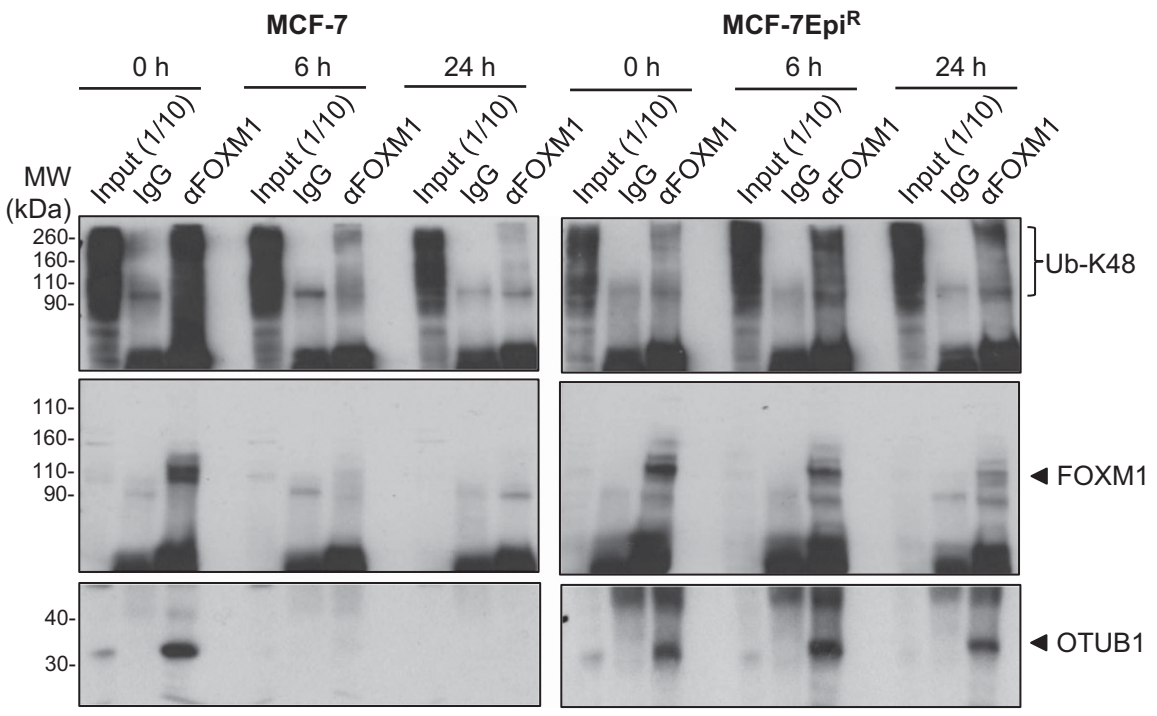

b
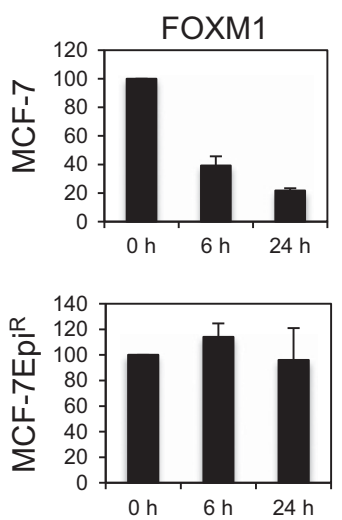

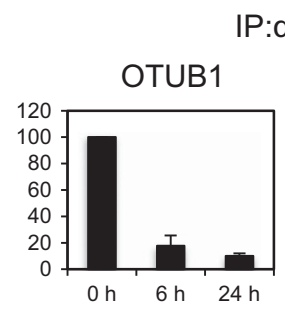

IP:aFOXM1
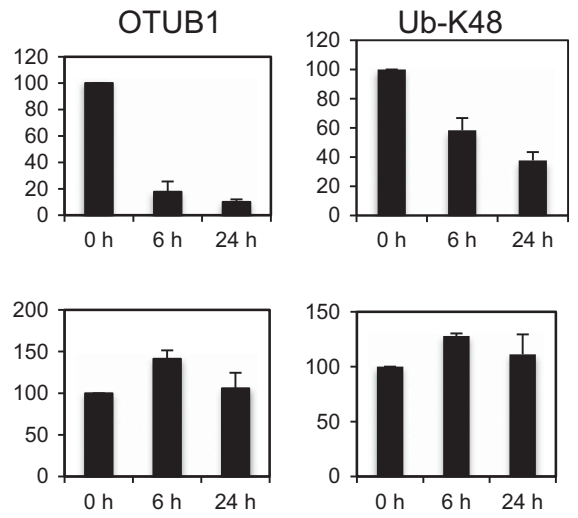
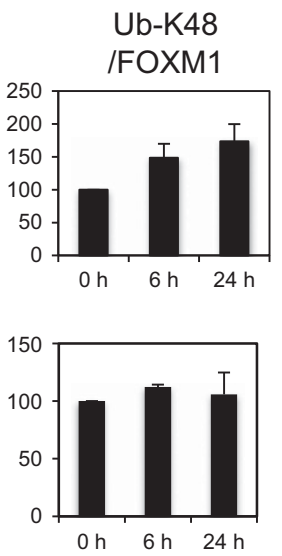

Figure 3. The downregulation of FOXM1 expression by epirubicin is associated with a decrease in OTUB1 binding and an increase in Lys48linked polyubiquitin conjugates in MCF-7 cells. (a) Protein lysates prepared from MCF-7 and cells at 0, 6 and $24 \mathrm{~h}$ following treatment with $1 \mu \mathrm{M}$ epirubicin were subjects to imnunoprecipitation with a FOXM1 antibody ( $\mathrm{FOOXM} 1$ ). The Input and immunoprecipitates were then analysed by western blot analysis using antibodies against Lys48-linked ubiquitin, OTUB1 and FOXM1. Representative co-immunprecipitation results are shown. (b) The Lys48-linked polyubiquitin, OTUB1 and FOXM1 images were quantified using ImageJ analysis and plotted against signals at $0 \mathrm{~h}$. The ratios of Lys48-polyubiquitin- conjugated FOXM1 relative to FOXM1 precipitates were also shown. Data shown represent the mean \pm s.d. from three independent experiments. ( $t$-test: $6 \mathrm{~h}$ or $24 \mathrm{~h}$ versus $0 \mathrm{~h}$ epirubicin treatment; ${ }^{*}$ significant $P<0.05,{ }^{* *}$ very significant $P<0.01$, and ns: not significant). ANOVA analysis was also performed on these data with post hoc test (Supplementary Figure S4).

MCF-7 with either the empty expression vector, wild-type (WT) OTUB1, or the OTUB1(C91S) mutant and studied the levels of K48polyubiquitin chains associated with FOXM1 by immunoprecipitation (Figure4c). The FOXM1 co-immunoprecipitation analysis showed that overexpression of OTUB1, but not the OTUB1(C91S) mutant, substantially depleted the levels of K48-polyubiquitin chains and induced FOXM1 expression, particularly following epirubicin treatment (Figure $4 \mathrm{c}$ ). These results suggest that the deubiquitinase activity of OTUB1 is required for the suppression of Lys48-linked FOXM1 polyubiquitination chains and for enhancing FOXM1 stability.

OTUB1 enhances FOXM1 stability in response to epirubicin treatment

To examine the role of OTUB1 in regulating FOXM1 protein stability, MCF-7 cells were transfected with either control vector, OTUB1(WT) or OTUB1 (C91S, and subjected to treatment with epirubicin and then the translation inhibitor cycloheximide (Figure 5). Inhibition of de novo protein synthesis by cycloheximide caused a decline in FOXM1 protein levels in MCF-7 cells treated with epirubicin. Under these conditions, the rate of FOXM1 loss was reduced in MCF-7 cells transfected with OTUB1(WT) compared with cells transfected with the empty vector control. By contrast, the rates for the decline in FOXM1 levels were similar in MCF-7 cells transfected with the OTUB1 (C91S) mutant and the empty vector. These findings indicate that the degradation of FOXM1 is impaired by overexpression of WT OTUB1 but not a mutant that lacks catalytic activity. Conversely, knockdown of OTUB1 by siRNA smart pool resulted in decreased half-life of FOXM1 in MCF-7 cells treated with epirubicin compared with cells transfected with non-silencing control siRNA. Together, these results suggest that the suppression of FOXM1 degradation by OTUB1 in response to epirubcin requires its deubiquitinating catalytic activity, further confirming that FOXM1 is a novel target of the deubiquitinase OTUB1. As an internal control, we measured the turnover of cyclin B1, a target of FOXM1 and not OTUB1, in response to epirubicin treatment. Taken together, the results provided strong evidence that OTUB1 suppresses FOXM1 ubiquitination and degraduation. 


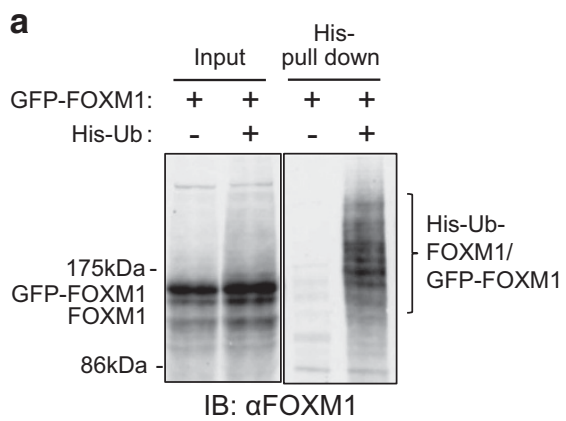

b

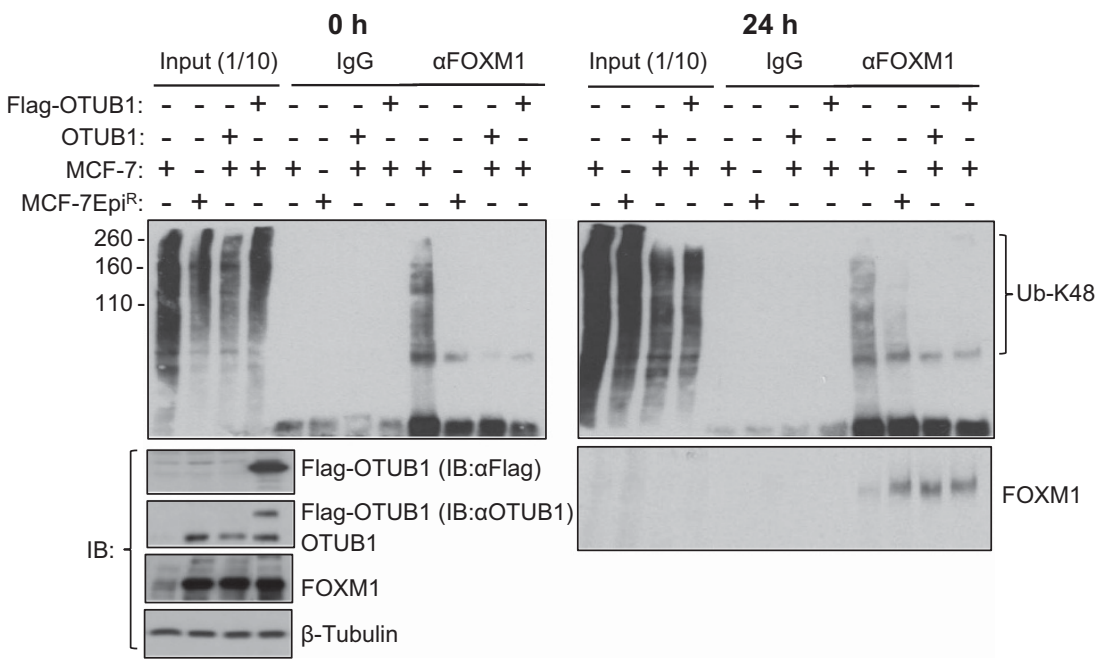

C

$\mathrm{Oh}$

$24 \mathrm{~h}$

pcDNA3: $\frac{\text { Input (1/10) }}{+--} \frac{\lg G}{+--+--} \frac{\text { aFOXM1 }}{+--} \frac{\text { Input (1/10) }}{+--+--}$

OTUB1: - + - - + - + -

OTUB1(C91S): - _ + - _ + + +

$-+-\ldots+-{ }_{-}+$
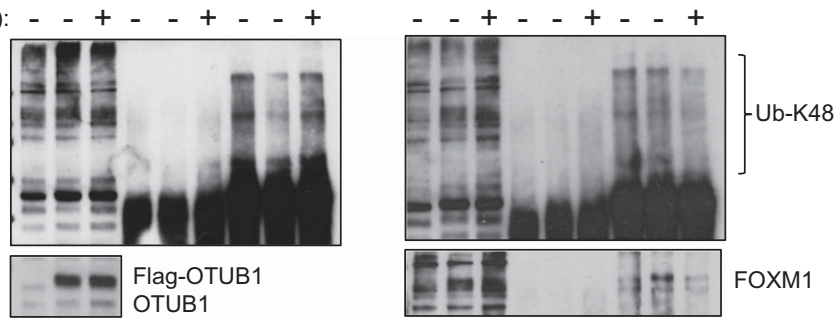

IB:

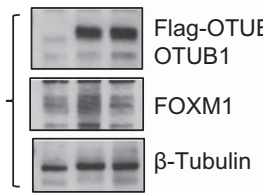

Figure 4. FOXM1 is modified by ubiquitination and its Lys48-linked polyubiquitin conjugates can be repressed by OTUB1. (a) MCF-7 cells were co-transfected with eGFP-FOXM1 and His-Ubiquitin. Ubiquitinated proteins were purified using $\mathrm{Ni}^{2+}$-column affinity pull-down under denaturing conditions (guanidinium chloride). Input and His-tagged Ubiquitin pulled-down proteins were probed for FOXM1 using an antiFOXM1 antibody, and polyubiquitinated FOXM1 was detectable, demonstrating the covalent linkage of His-Ubiquitin to FOXM1. (b) MCF-7, MCF-7Epi ${ }^{\mathrm{R}}$, and OTUB1 and Flag-OTUB1 stably expressing MCF-7 cells were treated with $1 \mu \mathrm{m}$ epirubicin for $0 \mathrm{~h}$ or $24 \mathrm{~h}$ and $1 \mu \mathrm{M} \mathrm{MG} 132 \mathrm{for} 6 \mathrm{~h}$ and then subjected to immunoprecipitation with an FOXM1 antibody. Inputs (1/10 of IP) and IP products with control IgG and the FOXM1 antibody were resolved on western blot and probed for Lys48-linked polyubiquitin conjugates and FOXM1. The Inputs were also immunoblotted for FOXM1, OTUB1, $\beta$-tubulin and Flag-OTUB1 (anti-Fiag or $\alpha$ Flag) with the respective antibodies. (c) MCF-7 cells transfected with control pcDNA3, Flag-OTUB1(WT) or the Flag-tagged catalytically dead mutant OTUB1(C91S) were treated with $1 \mu \mathrm{m}$ epirubicin for $0 \mathrm{~h}$ or $24 \mathrm{~h}, 10 \mu \mathrm{m}$ MG132 for $6 \mathrm{~h}$ and then subjected to immunoprecipitation with an FOXM1 antibody and western blotted with specific antibodies as in (c).

OTUB1 promotes cell proliferation and epirubicin resistance through targeting FOXM1

Recent evidence demonstrates that FOXM1 can enhance cancer cell proliferation and protect cells from genotoxic agent-induced cell death by enhancing DNA damage repair. ${ }^{5,8,12}$ To test the possible function of OTUB1 in the regulation of breast cancer cell proliferation and DNA-damaging agent resistance, we transiently transfected MCF-7 cells with an OTUB1 expression plasmid and OTUB1 siRNA pool, and studied their effects on MCF-7 cell proliferation. The result showed that overexpression of OTUB1 

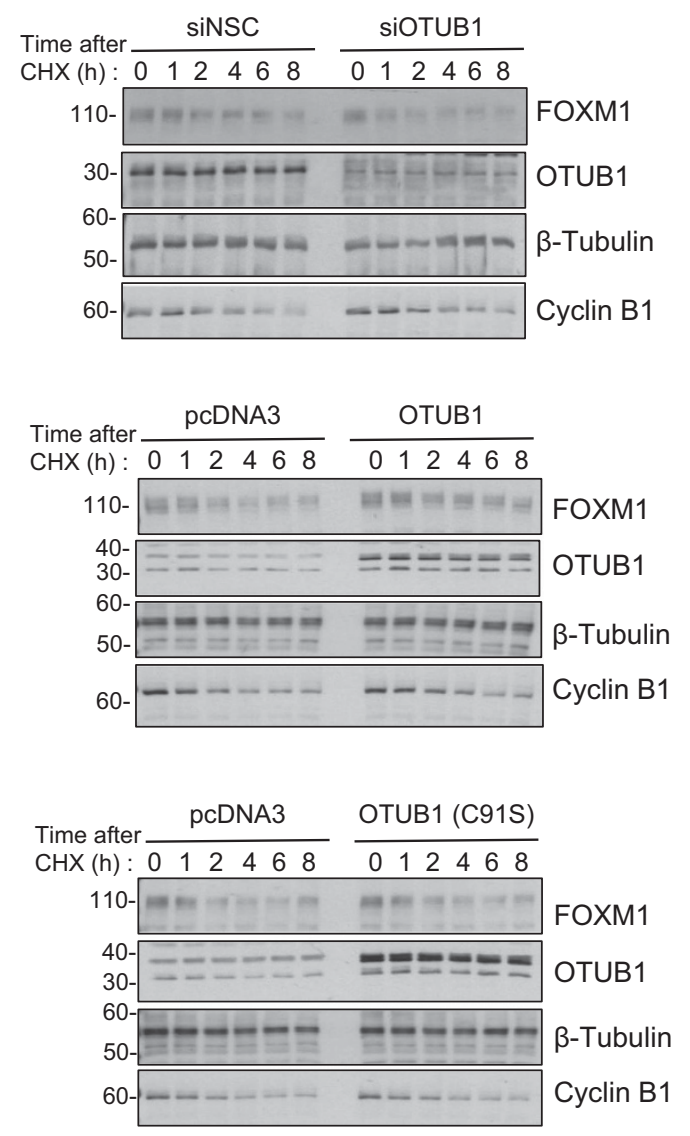
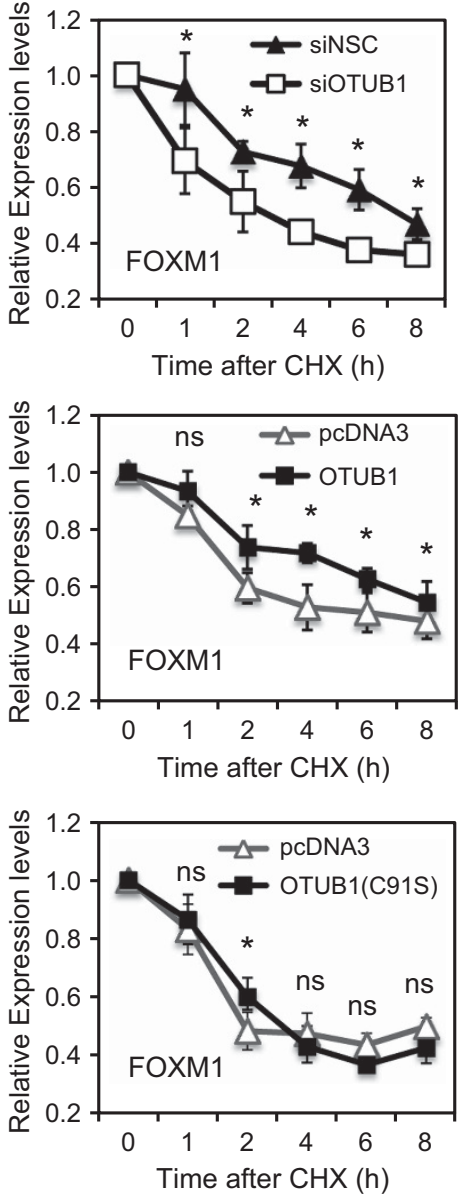

Figure 5. OTUB1 supresses the degradation of FOXM1 in epirubicin-treated MCF-7 cells. (a) MCF-7 cells transfected with control non-silencing siRNA or Smart Pool siRNA targeting OTUB1 were treated with $1 \mu \mathrm{m}$ epirubicin for $16 \mathrm{~h}$. These epirubicin-treated MCF-7 cells were then treated with cycloheximide, and protein lysates prepared from 0 to $8 \mathrm{~h}$ following cyclohexamide treatment. Protein expression levels of FOXM1, OTUB1, $\beta$-tubulin and cyclin B1 in these MCF-7 lysates were examined by western blotting. Densitometry was used to quantify the FOXM1 and $\beta$-tubulin levels from which independent background readings were subtracted. Western blots are representative of three independent experiments. The relative expression levels shown (right panels) are means \pm s.e.m. of the ratios of FOXM1 to $\beta$-tubulin levels relative to those at $0 \mathrm{~h}$. Statistical significance was determined by Student's $t$-test $(* P \leqslant 0.05$, significant; ns, non-significant). In (b) and (c), MCF-7 cells transfected with control pcDNA3 and (b) Flag-OTUB1(WT) or (c) the mutant Flag-OTUB1(C91S) were treated and analysed as in (a).

significantly enhanced MCF-7 cell proliferation, whereas OTUB1 depletion decreased the rates of proliferation of MCF-7 cells (Supplementary Figure S7). Furthermore, we also found that OTUB1 overexpression enhanced the resistance of MCF-7 cells to epirubicin (Supplementary Figure S7). Conversely, OTUB1 silencing potentiated the anti-proliferative function of epirubicin in MCF-7 cells (Supplementary Figure S7). We next tested whether FOXM1 has $a$ role in the cell-proliferative and epirubicin-resistant functions of OTUB1. To this end, we transfected WT and Foxm $1^{-1-}$ mouse embryo fibroblasts (MEFs) with either the empty expression vector, OTUB1(WT), or the OTUB1(C91S) mutant and examined their effects on cell proliferation and epirubicin resistance by SRB assay. Overexpression of OTUB1, but not the OTUB1(C91S) mutant, significantly enhanced the cell proliferation as well as the viability of WT MEFs in response to epirubicin (Figure 6). By contrast, there were no significant differences in cell proliferation rates and epirubicin sensitivity in FOXM1-deficent MEFs transfected with vector control, OTUB1 and the OTUB1 (C91S) mutant. In addition, clonogenic assays showed that at 0, 20 and $40 \mathrm{nM}$ epirubicin, the colony formation capacity of WT MEFs transfected with WT OTUB1 was significantly enhanced when compared with MEFs transfected with empty expression vector or the OTUB1(C91S) mutant (Figure 7). Similar to the cell proliferation data, there were no appreciable differences in clonogenicity in Foxm $1^{-/-}$MEFs transfected with empty vector control, WT OTUB1 and the mutant OTUB1(C91S). These results indicate that FOXM1 is a key target of OTUB1 and that FOXM1 mediates the oncogenic and DNA damage resistant functions of OTUB1.

Correlation between OTUB1 and FOXM1 expression in breast cancer patient samples

To establish further the physiological significance and clinical relevance of the regulation of FOXM1 by OTUB1 in breast cancer, FOXM1 and OTUB1 expression was assessed by immunohistochemistry in 116 breast cancer patient samples (Figure 8). OTUB1 was predominantly expressed in cytoplasm, consistent with its function as a deubiquitinating enzyme. Immunohistochemical analysis results showed that OTUB1 expression significantly correlated with FOXM1 expression (Chi-square test, $P=0.034$ ). There was also significant correlation between OTUB1 and oestrogen receptor (ER)a observed in our cohort (Supplementary Figure S8), consistent with the previous finding that OTUB1 could deubiquitinate and inhibit the degradation of ERa. ${ }^{18}$ However, there were no significant correlations between OTUB1 and other clinicopathological parameters including progesterone status, 
a
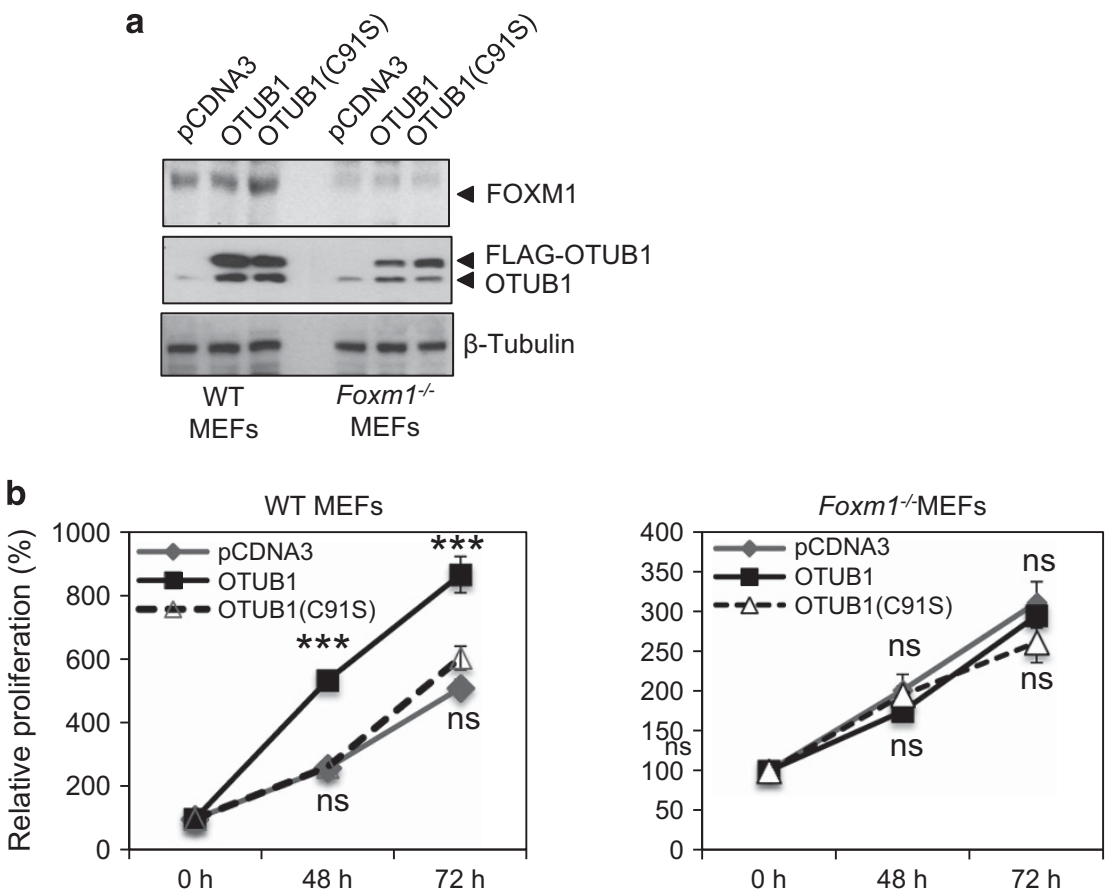

C

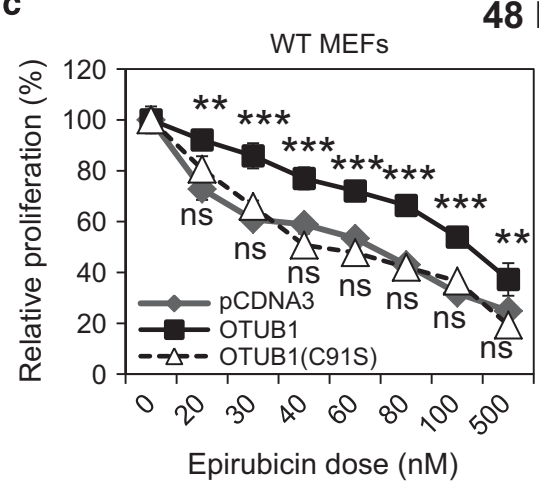

$48 \mathrm{~h}$ Epirubicin
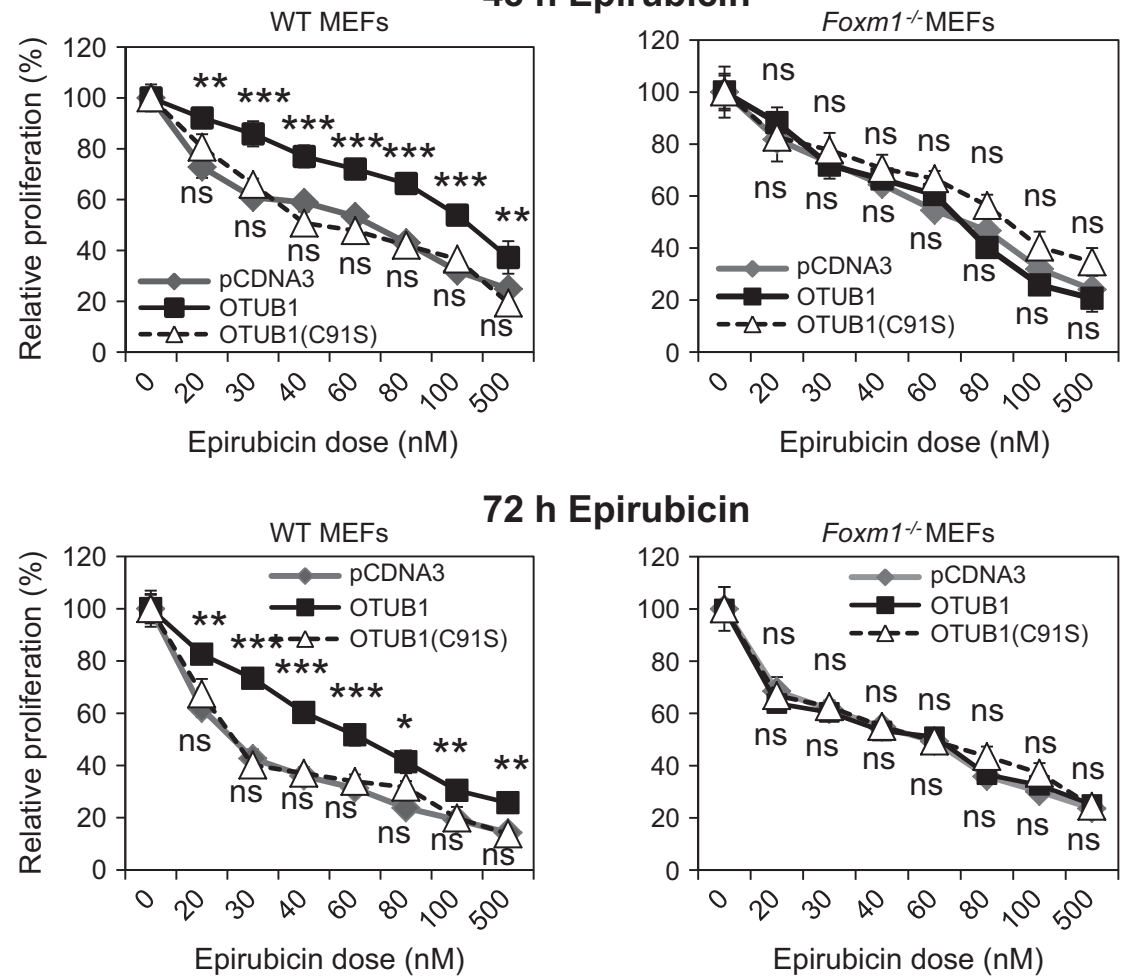

Figure 6. Overexpression of OTUB1 promotes cell proliferation, and epirubicin resistance in WT but not Foxm1-deficent MEFs. WT and Foxm1-deficent MEFs were transiently transfected with either the control pCDNA3 and Flag-OTUB1(WT) or the mutant Flag-OTUB1(C91S). (a) Twenty-four hours after transfection, protein lysates were prepared from these cells and then analysed for the expression of FOXM1, OTUB1 and $\beta$-Tubulin. (b) Twenty-four hours after transfection, aliquots of the transfected cells were split into 96 well plates and their proliferation analysed at the times indicated by SRB assays. Cell proliferation assays revealed that while untreated WT MEFs cells transiently transfected with Flag-OTUB1(WT) but not Flag-OTUB1(C91S), proliferated faster than the control pcDNA3-Flag cells, there was no difference in the Foxm1-deficent MEFs (c) The transfected cells were also treated with a range of doses of epirubicin (0-500 nM) and their proliferative rates assayed by SRB assay at 48 and $72 \mathrm{~h}$ after epirubicin treatment. Statistical significance was determined by Student's $t$-test $\left(* P \leqslant 0.05,{ }^{* *} P \leqslant 0.01\right.$, ${ }^{* * *} P \leqslant 0.005$; ns, non-significant) by comparing the proliferation rates of cells transfected with Flag-OTUB1(WT) or Flag-OTUB1(C91S) with the control pcDNA3-Flag transfected cells. 
a

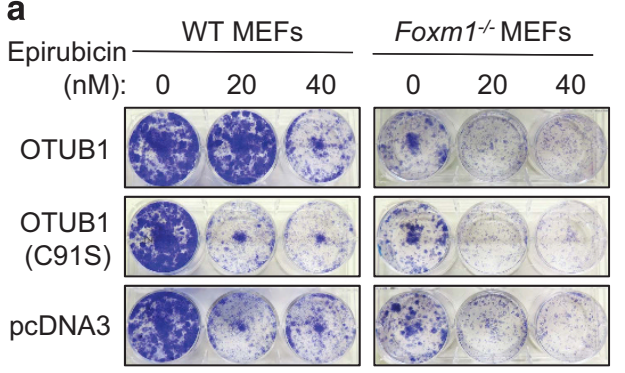

b

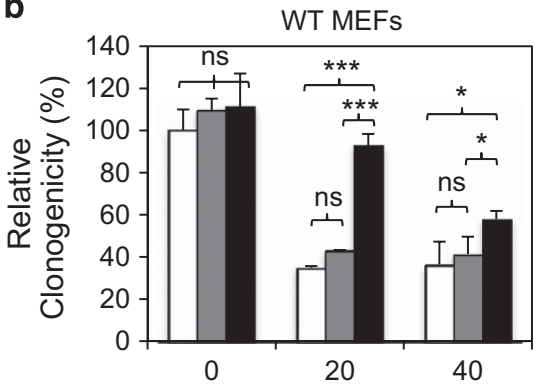

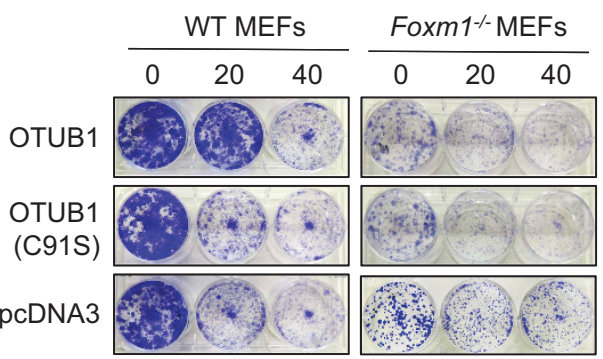

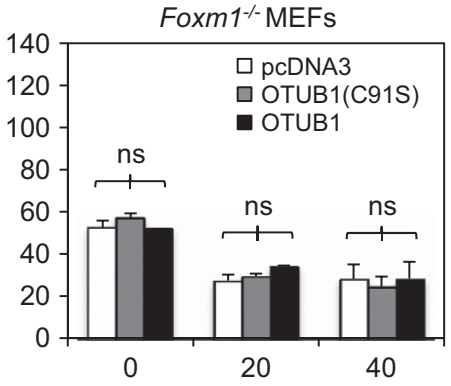

Epirubicin (nM)

Figure 7. Overexpression of OTUB1 promotes clonogenicity in WT but not Foxm1-deficent MEFs. WT and Foxm1-deficent MEFs were transfected with either the control pcDNA3 and Flag-OTUB1(WT) or the mutant Flag-OTUB1(C91S). Twenty-four hours after transfection, the 2000 cells were seeded in six-well plates, treated with 0, 20 or $40 \mathrm{~nm}$ of epirubicn, grown for 15 days and then stained with crystal violet (top panel). The result (bottom panel) represents the average of three independent experiments \pm s.d. Statistical significance was determined by Student's $t$-test $\left({ }^{*} P \leqslant 0.05,{ }^{* * *} P \leqslant 0.005 ; \mathrm{ns}\right.$, non-significant).

histological type, lymph node involvement and tumour stage (Supplementary Figure S8). Intriguingly, survival analysis showed no significant correlation between OTUB1 expression and patients' survival (Supplementary Figure S9). In agreement with our survival data, analysis of OTUB1 transcript expression in a previously published cohort (3455 breast cancer patients) ${ }^{19}$ also revealed that OTUB1 mRNA expression level was not significantly associated with poor survival $(P=0.42$ for overall survival, Kaplan-Meier analysis) (Supplementary Figure S10). However, in another published cohort of 1926 cases of lung cancer, ${ }^{19}$ OTUB1 mRNA expression level was a significant poor prognostic marker ( $P=0.0025$ for overall survival, Kaplan-Meier analysis) (Supplementary Figure S11). The discrepancy between breast cancer and other cancer types is likely because OTUB1 substrates, in particular $\mathrm{ERa}$, have a particularly essential role in breast cancer initiation, progression and treatment. Although ERa signalling promotes breast cancer proliferation, expression of ERa is also associated with good prognosis because these patients are likely to benefit from endocrine therapy. Moreover, in our tissue microarray cohort, tumour stage and lymph node involvement were significantly associated with poor survival $(P=0.007$ and $P=0.017$, respectively, for overall survival; $P=0.001$ and $P=0.01$, respectively, for disease-specific survival, refer to cox regression univariate analysis table) (Supplementary Figures S11 and S12). Therefore, to remove the interferences of the clinicopathological parameters on the survival analysis of OTUB1, multivariate analyses using Cox regression model were performed. In multivariate analysis, OTUB1 expression became significantly associated with poor survival after being adjusted for the clinicopathological parameters, including ER status, progesterone status, tumour stage, histological type and lymph-node involvement $(P=0.033$, relative risk $=2.859$ for overall survival and $P=0.013$, relative risk $=4.048$ for disease-specific survival) (Supplementary Figure S11). In this cohort, $60 \%$ of patients received chemotherapy. For this subgroup of patients, elevated OTUB1 was also significantly associated with poor survival after being adjusted for all the clinicopathological parameters $(P=0.032$, relative risk $=3.822$ for overall survival and $P=0.032$, relative risk $=3.822$ for diseasespecific survival) (Supplementary Figure S12). As resistance to chemotherapy is known to be associated with poorer survival, the results from cox regression analysis supported a role of OTUB1 in chemotherapy resistance. This is consistent with our previous study showing that FOXM1 overexpression confers resistance to genotoxic chemotherapy. ${ }^{9}$ Collectively, these data suggest that OTUB1 overexpression is associated with chemotherapeutic drug resistance, and this is likely to be mediated by promoting FOXM1 protein expression through supressing its ubiquitination and degradation.

\section{DISCUSSION}

FOXM1 has an essential function in enhancing DNA damage repair and genotoxic agent resistance. ${ }^{5,8-10}$ Previous research has also demonstrated that OTUB1 can modulate DNA damage response by promoting protein deubiquitination. ${ }^{17}$ We show, in here, that OTUB1 can promote deubiquitination and stabilization of FOXM1 in response to the genotoxic agent epirubicin in breast cancer.

Here, we also report that FOXM1 is conjugated with Lys48linked polyubiquitin chains. FOXM1 expression is downregulated in response to epirubicin treatment in MCF-7 cells. This finding is consistent with the established concept that proteins with Lys48linked polyubiquitin chains are generally targeted for proteasomedependent degradation. ${ }^{20-27}$ Different OTU family proteins have certain specificity towards distinct types of ubiquitin linkages. ${ }^{28}$ For example, OTUB1 preferentially targets polyubiquitin chains joined by Lys48 bonds, while OTUB2 has specificity towards chains associated through Lys63. ${ }^{28}$

In concordance, our data demonstrate that OTUB1 overexpression represses the conjugation of Lys48-linked polyubiquitin chains on FOXM1 and impairs its degradation, thus promoting resistance to genotoxic agents. Our previous study shows that SUMOylation inhibits FOXM1 activity, promotes its ubiquitination 
a
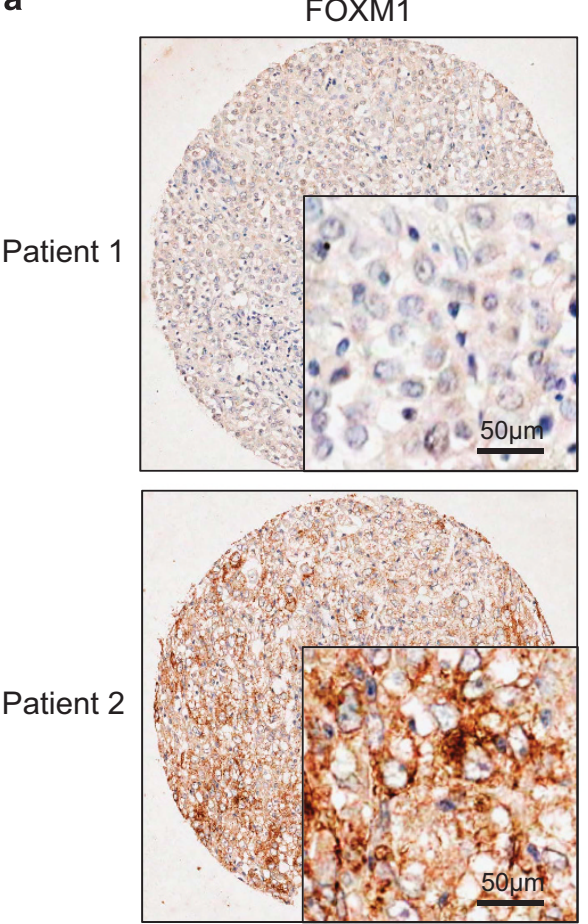

OTUB1
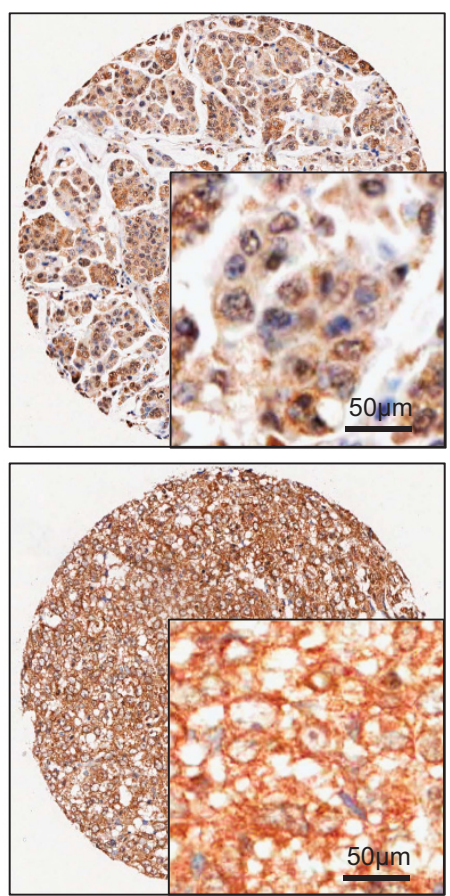

b

FOXM1* OTUB1 Crosstabulation

Count

\begin{tabular}{|ll|c|c|c|}
\hline & & \multicolumn{2}{|c|}{ OTUB1 } & \\
& & 0.00 & 1.00 & Total \\
\hline FOXM1 & 0.00 & 30 & 25 & 55 \\
& $\overline{1} 1.00$ & 17 & 33 & 50 \\
Total & & 47 & 58 & 105 \\
\hline
\end{tabular}

Chi-Square Tests

\begin{tabular}{|c|c|c|c|c|c|}
\hline & Value & df & $\begin{array}{l}\text { Asymp. Sig. } \\
\text { (2-sided) }\end{array}$ & $\begin{array}{l}\text { Exact Sig. } \\
\text { (2-sided) }\end{array}$ & $\begin{array}{l}\text { Exact Sig. } \\
\text { (1-sided) }\end{array}$ \\
\hline Pearson Chi-Square & $4.471^{\mathrm{a}}$ & 1 & 0.034 & \multirow{6}{*}{0.049} & \multirow{6}{*}{0.027} \\
\hline Continuity Correction ${ }^{\mathrm{b}}$ & 3.679 & 1 & 0.055 & & \\
\hline Likelihood Ratio & 4.512 & 1 & 0.034 & & \\
\hline Fisher's Exact Test & \multirow{3}{*}{$\begin{array}{c}4.429 \\
105\end{array}$} & \multirow{3}{*}{1} & \multirow{3}{*}{0.035} & & \\
\hline $\begin{array}{l}\text { Linear-by-Linear } \\
\text { Association }\end{array}$ & & & & & \\
\hline $\mathrm{N}$ of Valid Cases & & & & & \\
\hline
\end{tabular}

a. 0 cells $(0.0 \%)$ have expected count less than 5 . The minimum expected count is 22.38 .

b. Computed only for a $2 \times 2$ table

Figure 8. Positive correlation between FOXM1 and OTUB1 expression in breast cancer patients. (a) FOXM1 and OTUB1 expression was assessed by immunohistochemistry using tissue microarray constructed from 116 breast cancer patient samples. OTUB1 was expressed predominantly in the cytoplasm. Representative staining images of one patient with high FOXM1 and OTUb1 expression and one with low expression are shown. Images (magnification $\times 20$; Insets (magnification $\times 100$ ). Positive correlation between FOXM1 and OTUB1 expression was observed. (b) KIF20A staining were detected in both nuclear and cytoplasmic compartments and were correlated with FOXM1 staining. Statistical analysis revealed that OTUB1 were significantly correlated with FOXM1 expression $(P=0.034$, Chi-Square test).

and enhances its cytoplasmic translocation as well as degradation. ${ }^{10}$ Thus, deubiquitination of FOXM1 may not only result in the stabilization of FOXM1 but may also promote its nuclear retention. $^{10}$

In addition to its function as a deubiquitinase, previous studies have shown that OTUB1 can suppress ubiquitination by inhibiting the E2 ubiquitin-conjugating enzymes independently of its catalytic activity. For example, OTUB1 suppresses RNF168-dependent polyubiquitination independently of its catalytic activity. Accordingly, OTUB1 binds to and inhibits UBC13 (UBE2N), the cognate E2 enzyme for RNF168. ${ }^{17}$ OTUB1 also suppresses UBCH5 E2 enzyme and stabilizes the $\mathrm{p} 53$ protein. ${ }^{14,29}$ However, we demonstrate here that OTUB1 suppresses the ubiquitination and degradation of FOXM1 through its deubiquitinase enzymatic activity. Our data show that WT OTUB1, but not the OTUB1(C91S) catalytic dead mutant, can enhance the stability of FOXM1 in response to 
epirubicin in MCF-7 cells, suggesting that the deubiquitinase activity is required for the degradation of FOXM1. The fact that OTUB1 interacts with FOXM1 also supports that FOXM1 deubiquitination occurs through a catalytic mechanism, that requires interactions with OTUB1.

The physiological relevance of the regulation of FOXM1 by OTUB1 is underscored by the strong and significant association between FOXM1 and OTUB1 in breast cancer patient samples. Despite not being an independent prognostic marker in KaplanMeier survival analysis, OTUB1 is significantly associated with poorer prognosis in multivariate regression analysis, particularly in breast cancer patients who have received chemotherapy. This argues that OTUB1 is likely to be linked to chemotherapy sensitivity in breast cancer. In agreement, the overexpression of OTUB1 has been shown to be associated with poorer outcome and cancer progression. For example, OTUB1 is associated with metastasis and is a predictive marker of poor prognosis in colorectal cancer. ${ }^{30,31}$ In addition, OTUB1 promotes tumourigenesis and cell invasion in prostate cancer. ${ }^{32}$

Kaplan-Meier survival analysis shows that OTUB1 expression is not correlated with clinical outcome in breast cancer patients, even though OTUB1 is a predictor for prognosis in patients with other malignancies, such as colorectal cancer. $^{30,31}$ Similarly, despite OTUB1 mRNA level being a prognostic marker for lung cancer patients, its expression has no predictive value for patients diagnosed with breast cancer. This is likely to be due to the complex role played by OTUB1 in breast cancer, as it regulates the expression and activity of a variety of substrate proteins with diverse and sometimes opposing functions in breast cancer initiation, progression and treatment. OTUB1 may also affect the stability of proteins, which contribute towards breast cancer progression and treatment. For example, OTUB1 has been reported to be an ERa-interacting protein. OTUB1 can suppress ubiquitination and stabilize the expression of ERa in the chromatin and thereby, negatively regulate its transcription. ${ }^{18}$ In agreement, we also found a significant positive correlation between OTUB1 expression and ER status in our study (Supplementary Figure S8). While ERa signalling has a key role in stimulating initial breast cancer cell proliferation during tumourigenesis, the expression of $\mathrm{ERa}$ is also an indication of a good outcome in breast cancer patients as ERa-positive patients can often be treated with endocrine therapy. ${ }^{33}$ Another OTUB1 target in breast cancer is the tumour suppressor $\mathrm{p} 53$. In response to DNA-damaging agents, OTUB1 can stabilize and activate p53, leading to the inhibition of cell proliferation and apoptosis. ${ }^{14,29}$ However, p53 expression and function is commonly lost during tumourigenesis and thus, the role of OTUB1 in the subsequent breast cancer progression and drug resistance will not be clear-cut. ${ }^{34,35}$

The finding that overexpression of OTUB1 has a prominent role in promoting cell proliferation and drug resistance suggests that OTUB1 is a potent oncogene. This is further supported by the fact that overexpression of OTUB1 is associated with poorer prognosis in many malignancies. ${ }^{30-32}$ However, except for ERa, few oncogenes have been identified to be substrates of OTUB1. ${ }^{18}$ In here, we not only show that the potent oncogene FOXM1 is a target of OTUB1 but also present evidence to demonstrate that the oncogenic and genotoxic functions of OTUB1 depend on the expression of a functional FOXM1, suggesting that OTUB1 promotes cell proliferation and epirubicin resistance predominantly through targeting FOXM1.

Collectively, these results provide strong evidence to suggest that not only OTUB1 deubiquitinase activity prevents the proteasomal degradation of FOXM1 upon epirubicin treatment, OTUB1 also regulates steady-state expression of FOXM1. Consistent with the emerging oncogenic role of OTUB1 in cancer, the proliferative and clonogenic assays also show that OTUB1 can enhance the proliferative rate and genotoxic agent resistance of breast cancer cells and that FOXM1 is a crucial substrate of OTUB1 in cancer cell proliferation and genotoxic agent resistance.

\section{MATERIALS AND METHODS}

Cell culture, plasmids and transfection reagents

The MCF-7 cell line used originated from the American Type Culture Collection and was acquired through CRUK cell bank (London, UK). MCF-7Epi ${ }^{R}$ cells and MEFs have previously been described. ${ }^{8,9}$ Cells were cultured in DMEM (Sigma-Aldrich, Poole, UK) supplemented with 10\% (v/v) feotal calf serum and $2 \mathrm{~mm}$ glutamine at $37^{\circ} \mathrm{C}$. Epirubicin Hydrochloride $(2 \mathrm{mg} / \mathrm{ml}(3.4 \mathrm{~mm})$ in $0.9 \%$ sodium chloride, Medac, Germany) was obtained from Imperial College Healthcare (London, UK). The pcDNA3-Flag-OTUB1 and -OTUB1(C91S) plasmids used in this study have previously been described. ${ }^{17}$ The eGFP-FOXM1 and pcDNA3-FOXM1 expression plasmids have also been described. ${ }^{10}$ For ubiquitination studies, cells were treated with $10 \mu \mathrm{M}$ MG132 (M7449; Sigma-Aldrich) for $6 \mathrm{~h}$ before collection for analysis. Cells were transfected using FuGENE 6 transfection reagent (Promega, Southampton, UK) and XtremeGENE HP reagent (Roche Diagnostics, Welwyn Garden City, UK) as recommended by the manufacturers.

\section{Ni-NTA pull-down assays}

His-tagged proteins were purified by nickel magnetic agarose beads (Qiagen, Manchester, UK) under denaturing conditions as described. ${ }^{36}$ For details, see also Supplementary Materials and Methods.

\section{Quantitative real-time PCR (qRT-PCR)}

Total RNA was extracted with the RNeasy Mini Kit (Qiagen). Complementary DNA generated by Superscript III reverse transcriptase and oligo-dT primers (Invitrogen, Paisley, UK) was analysed by qRT-PCR as described. ${ }^{8,12}$ See also Supplementary Materials and Methods.

\section{Gene silencing with siRNAs}

For gene silencing, cells were transiently transfected with siRNA SMARTpool reagents purchased from Thermo Scientific Dharmacon (Lafayette, CO, USA) using Oligofectamine (Invitrogen, Life Technologies Ltd, Paisley, UK) according to the manufacturer's instructions. siRNAs On Target Smart Pool used were: siRNA FOXM1 (L-009762-00), siRNA OTUB1 (L-021061-00) and the non-silencing control siRNA (D-001810-10-05).

\section{Measure of FOXM1 protein turnover}

The turnover rate of endogenous FOXM1 in MCF-7 cells was determined using cycloheximide (01810; Sigma-Aldrich) inhibition of protein synthesis. For details, see Supplementary Materials and Methods.

\section{Immunoprecipitation and western blotting}

Western blotting was performed on whole-cell extracts by lysing cells in buffer or precipitates in the presence of $\mathrm{N}$-ethyl-amide $(10 \mathrm{~mm})$ (Sigma UK, Poole, UK) as previously described. ${ }^{9,37}$ See Supplementary Materials and Methods for antibodies used.

\section{Clonogenic assay}

Clonogenic assay has been described. ${ }^{9}$ Also, see Supplementary Methods and Materials.

Tissue microarray, immunohistochemistry and staining scoring These reagents and analyses have been described. ${ }^{9}$ For details, see Supplementary Methods and Materials.

\section{Statistical analysis}

All statistics were determined using SPSS 16.0 and Windows XP, Excel (Imperial College London, Software Shop, UK). Also, see Supplementary Methods and Materials.

\section{CONFLICT OF INTEREST}

The authors declare no conflict of interest. 


\section{ACKNOWLEDGEMENTS}

EW-F Lam and AR Gomes were supported by grants from Cancer Research UK, EW-F Lam and S Zona by grants from Breast Cancer Campaign. U Karunarathna is the recipient of a BBSRC studentship. $\mathrm{P}$ Khongkow and $\mathrm{M}$ Kongsema were supported by grants from the Royal Thai Government Scholarship. US Khoo received funding from Committee on Research and Conference Grants from the University of Hong Kong (CRCG) (201007176118). We also thank for help with this work.

\section{REFERENCES}

1 Savarese DM, Hsieh C, Stewart FM. Clinical impact of chemotherapy dose escalation in patients with hematologic malignancies and solid tumors. J Clin Oncol 1997; 15: 2981-2995.

2 Myatt SS, Lam EW. The emerging roles of forkhead box (Fox) proteins in cancer. Nat Rev Cancer 2007; 7: 847-859.

3 Myatt SS, Lam EW. Targeting FOXM1. Nat Rev Cancer 2008; 8: 242.

4 Kwok JM, Myatt SS, Marson CM, Coombes RC, Constantinidou D, Lam EW. Thiostrepton selectively targets breast cancer cells through inhibition of forkhead box M1 expression. Mol Cancer Ther 2008; 7: 2022-2032.

5 Kwok JM, Peck B, Monteiro LJ, Schwenen HD, Millour J, Coombes RC et al. FOXM1 confers acquired cisplatin resistance in breast cancer cells. Mol Cancer Res 2010; 8: 24-34.

6 McGovern UB, Francis RE, Peck B, Guest SK, Wang J, Myatt SS et al. Gefitinib (Iressa) represses FOXM1 expression via FOXO3a in breast cancer. Mol Cancer Ther 2009; 8: 582-591.

7 Khongkow P, Gomes AR, Gong C, Man EP, Tsang JW, Zhao F et al. Paclitaxel targets FOXM1 to regulate KIF20A in mitotic catastrophe and breast cancer paclitaxel resistance. Oncogene 2016; 35; 990-1002.

8 Monteiro LJ, Khongkow P, Kongsema M, Morris JR, Man C, Weekes D et al. The Forkhead Box M1 protein regulates BRIP1 expression and DNA damage repair in epirubicin treatment. Oncogene 2013; 32: 4634-4645.

9 Khongkow P, Karunarathna U, Khongkow M, Gong C, Gomes AR, Yague E et al. FOXM1 targets NBS1 to regulate DNA damage-induced senescence and epirubicin resistance. Oncogene 2014; 33: 4144-4155.

10 Myatt SS, Kongsema M, Man CW, Kelly DJ, Gomes AR, Khongkow P et al. SUMOylation inhibits FOXM1 activity and delays mitotic transition. Oncogene 2014; 33: 4316-4329.

11 Khasraw M, Bell R, Dang C. Epirubicin: is it like doxorubicin in breast cancer? A clinical review. Breast 2012; 21: 142-149.

12 Millour J, de Olano N, Horimoto Y, Monteiro L, Langer JK, Aligue R et al. ATM and p53 regulate FOXM1 expression via E2F in breast cancer epirubicin treatment and resistance. Mol Cancer Ther 2011; 10: 1046-1058.

13 de Olano N, Koo CY, Monteiro L, Pinto PH, Gomes AR, Aligue R et al. The p38 MAPK-MK2 axis regulates E2F1 and FOXM1 expression after epirubicin treatment. Mol Cancer Res 2012; 10: 1189-1202.

14 Sun XX, Challagundla KB, Dai MS. Positive regulation of p53 stability and activity by the deubiquitinating enzyme Otubain 1. EMBO J 2012; 31: 576-592.

15 Edelmann MJ, Iphofer A, Akutsu M, Altun M, di Gleria K, Kramer HB et al. Structural basis and specificity of human otubain 1-mediated deubiquitination. Biochem $J$ 2009; 418: 379-390.

16 Wang T, Yin L, Cooper EM, Lai MY, Dickey S, Pickart CM et al. Evidence for bidentate substrate binding as the basis for the K48 linkage specificity of otubain 1. J Mol Biol 2009; 386: 1011-1023.

17 Nakada S, Tai I, Panier S, Al-Hakim A, lemura S, Juang YC et al. Non-canonical inhibition of DNA damage-dependent ubiquitination by OTUB1. Nature 2010; 466: $941-946$.

18 Stanisic V, Malovannaya A, Qin J, Lonard DM, O'Malley BW. OTU Domaincontaining ubiquitin aldehyde-binding protein 1 (OTUB1) deubiquitinates estrogen receptor (ER) alpha and affects ERalpha transcriptional activity. J Biol Chem 2009; 284: 16135-16145.

19 Gyorffy B, Lanczky A, Eklund AC, Denkert C, Budczies J, Li Q et al. An online survival analysis tool to rapidly assess the effect of 22,277 genes on breast cancer prognosis using microarray data of 1,809 patients. Breast Cancer Res Treat 2010; 123: 725-731.

20 Chau V, Tobias JW, Bachmair A, Marriott D, Ecker DJ, Gonda DK et al. A multiubiquitin chain is confined to specific lysine in a targeted short-lived protein. Science 1989; 243: 1576-1583.

21 Deshaies RJ, Joazeiro CA. RING domain E3 ubiquitin ligases. Annu Rev Biochem 2009; 78: 399-434.

22 Finley D. Recognition and processing of ubiquitin-protein conjugates by the proteasome. Annu Rev Biochem 2009; 78: 477-513.

23 Xu P, Duong DM, Seyfried NT, Cheng D, Xie Y, Robert J et al. Quantitative proteomics reveals the function of unconventional ubiquitin chains in proteasomal degradation. Cell 2009; 137: 133-145.

24 Komander D, Rape M. The ubiquitin code. Annu Rev Biochem 2012; 81: 203-229.

25 Cohen MM, Amiott EA, Day AR, Leboucher GP, Pryce EN, Glickman MH et al. Sequential requirements for the GTPase domain of the mitofusin Fzo1 and the ubiquitin ligase SCFMdm30 in mitochondrial outer membrane fusion. J Cell Sci 2011; 124: 1403-1410.

26 Lipkowitz S, Weissman AM. RINGs of good and evil: RING finger ubiquitin ligases at the crossroads of tumour suppression and oncogenesis. Nat Rev Cancer 2011; 11: 629-643.

27 Weissman AM, Shabek N, Ciechanover A. The predator becomes the prey: regulating the ubiquitin system by ubiquitylation and degradation. Nat Rev Mol Cell Biol 2011; 12: 605-620.

28 Mevissen TE, Hospenthal MK, Geurink PP, Elliott PR, Akutsu M, Arnaudo N et al. OTU deubiquitinases reveal mechanisms of linkage specificity and enable ubiquitin chain restriction analysis. Cell 2013; 154: 169-184.

29 Li Y, Sun XX, Elferich J, Shinde U, David LL, Dai MS. Monoubiquitination is critical for ovarian tumor domain-containing ubiquitin aldehyde binding protein 1 (Otub1) to suppress UbcH5 enzyme and stabilize p53 protein. J Biol Chem 2014; 289: 5097-5108.

30 Zhou Y, Wu J, Fu X, Du W, Zhou L, Meng X et al. OTUB1 promotes metastasis and serves as a marker of poor prognosis in colorectal cancer. Mol Cancer 2014; 13: 258.

31 Liu X, Jiang WN, Wang JG, Chen H. Colon cancer bears overexpression of OTUB1. Pathol Res Pract 2014; 210: 770-773.

32 Iglesias-Gato D, Chuan YC, Jiang N, Svensson C, Bao J, Paul I et al. OTUB1 de-ubiquitinating enzyme promotes prostate cancer cell invasion in vitro and tumorigenesis in vivo. Mol Cancer 2015; 14: 8.

33 Fox EM, Davis RJ, Shupnik MA. ERbeta in breast cancer--onlooker, passive player, or active protector? Steroids 2008; 73: 1039-1051.

34 Malkin D, Li FP, Strong LC, Fraumeni JF Jr., Nelson CE, Kim DH et al. Germ line p53 mutations in a familial syndrome of breast cancer, sarcomas, and other neoplasms. Science 1990; 250: 1233-1238.

35 Singh S, Simon M, Meybohm I, Jantke I, Jonat W, Maass $\mathrm{H}$ et al. Human breast cancer: frequent p53 allele loss and protein overexpression. Hum Genet 1993; 90: 635-640.

36 Salghetti SE, Kim SY, Tansey WP. Destruction of Myc by ubiquitin-mediated proteolysis: cancer-associated and transforming mutations stabilize Myc. EMBO J 1999; 18: 717-726.

37 Hui RC, Francis RE, Guest SK, Costa JR, Gomes AR, Myatt SS et al. Doxorubicin activates $\mathrm{FOXO} 3 \mathrm{a}$ to induce the expression of multidrug resistance gene ABCB1 (MDR1) in K562 leukemic cells. Mol Cancer Ther 2008; 7: 670-678.

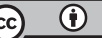

This work is licensed under a Creative Commons Attribution 4.0 International License. The images or other third party material in this article are included in the article's Creative Commons license, unless indicated otherwise in the credit line; if the material is not included under the Creative Commons license, users will need to obtain permission from the license holder to reproduce the material. To view a copy of this license, visit http://creativecommons.org/licenses/ by/4.0/

Supplementary Information accompanies this paper on the Oncogene website (http://www.nature.com/onc) 